\title{
Quantum and Classical Relaxation in the Proton Glass
}

\author{
Yejun Feng, ${ }^{1}$ C. Ancona-Torres, ${ }^{1}$ T. F. Rosenbaum, ${ }^{1, *}$ G. F. Reiter, ${ }^{2}$ D. L. Price, ${ }^{3}$ and E. Courtens ${ }^{4}$ \\ ${ }^{1}$ The James Franck Institute and Department of Physics, The University of Chicago, Chicago, Illinois 60637, USA \\ ${ }^{2}$ Physics Department, University of Houston, Houston, Texas 77204, USA \\ ${ }^{3}$ Centre de Recherches sur les Matériaux à Haute Temperature, CNRS, Orléans, France \\ ${ }^{4}$ LCVN, UMR 5587 CNRS, Université Montpellier II, F-34095 Montpellier, France
}

(Received 4 April 2006; published 4 October 2006)

\begin{abstract}
The hydrogen-bond network formed from a crystalline solution of ferroelectric $\mathrm{RbH}_{2} \mathrm{PO}_{4}$ and antiferroelectric $\mathrm{NH}_{4} \mathrm{H}_{2} \mathrm{PO}_{4}$ demonstrates glassy behavior, with proton tunneling the dominant mechanism for relaxation at low temperature. We characterize the dielectric response over seven decades of frequency and quantitatively fit the long-time relaxation by directly measuring the local potential energy landscape via neutron Compton scattering. The collective motion of protons rearranges the hydrogen bonds in the network. By analogy with vortex tunneling in superconductors, we relate the logarithmic decay of the polarization to the quantum-mechanical action.
\end{abstract}

DOI: 10.1103/PhysRevLett.97.145501

PACS numbers: 61.43.Fs, 66.35.+a, 77.22. $-\mathrm{d}$, 77.84.Fa

Glasses become trapped in a restricted set of local configurations and evolve extraordinarily slowly over time. Delineating the relationship between the structure and the dynamics is a critical element in establishing the essential nature of the glassy state, but this correspondence remains difficult to access in most systems. The proton glass [1], a structural analogue to magnetic spin glasses [2] with competing ferroelectric (FE) and antiferroelectric (AFE) order, is different. By combining dielectric spectroscopy over seven decades in frequency with a direct mapping of the real-space energy potential via neutron Compton scattering [3], we are able to describe quantitatively the highly choreographed proton dynamics within a hydrogen-bond network. Proton tunneling controls correlations between neighboring hydrogen bonds and dominates the long-time relaxation.

The hydrogen bond plays an important role in physics, chemistry, and biology [4], but it is probably most familiar from ice, where the freedom of different static configurations to accommodate Pauling's rule generates a macroscopic ground state and extra entropy contributing to the latent heat [5]. The hydrogen-bond network formed by Pauling's rule exists as well in other crystalline materials, for example, the piezoelectric $\mathrm{KH}_{2} \mathrm{PO}_{4}$ (KDP) family $[1,6]$. Protons in four O-H-O bonds attached to each $\left(\mathrm{PO}_{4}\right)^{3-}$ group are constrained to maintain singly charged $\left(\mathrm{H}_{2} \mathrm{PO}_{4}\right)^{-}$groups. Six distinct electrical dipole moments along three orthogonal axes can be formed with different Slater configurations [6]. While pure phases of $\mathrm{RbH}_{2} \mathrm{PO}_{4}$ (RDP) and $\mathrm{NH}_{4} \mathrm{H}_{2} \mathrm{PO}_{4}$ (ADP) are FE and AFE, respectively, at low temperature, a solid state solution creates a dipolar glass, $\mathrm{Rb}_{1-x}\left(\mathrm{NH}_{4}\right)_{x} \mathrm{H}_{2} \mathrm{PO}_{4}$ (RADP:100x), for concentrations $0.22<x<0.74$ [1]. The disorder, combined with the "frustration" arising from the competition between FE ordering along the $c$ axis in RDP and AFE ordering within the $a b$ plane for ADP, leads to the characteristic broad and frequency-dependent peak in the dielec- tric susceptibility vs temperature $T[1,2]$ and no more than short-range structural correlations [7]. The proton's pivotal role in relaxation is confirmed by the strong isotope effect on low $T$ thermal properties [8].

The dielectric spectroscopy is performed for $0.36 \mathrm{~K} \leq$ $T \leq 300 \mathrm{~K}$ by the combined use of ${ }^{3} \mathrm{He}$ and helium-flow cryostats, with overlap of data sets for $T=2.5-5 \mathrm{~K}$. Detailed frequency $f$ scans from $0.4 \mathrm{~Hz}$ to $2.5 \mathrm{MHz}$ were taken at $\mathrm{T} \leq 30 \mathrm{~K}$, where the glassy behavior is manifest.

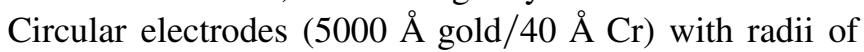
3-4 mm were evaporated concentrically on both sides of 1-mm thick $a c$-cut RADP:72 and RADP:35 single crystal plates, holding the temperature at the sample surface below $100{ }^{\circ} \mathrm{C}$ to prevent decomposition during evaporation. A HP4192A LCR meter was used to cover measurements from $5 \mathrm{kHz}$ to $2.5 \mathrm{MHz}$ and SR830 lock-in amplifiers were used from $0.4 \mathrm{~Hz}$ to $10 \mathrm{kHz}$. The imaginary part of the dielectric susceptibility is approximately 20 times smaller than the real part at all $f$ and $T$ reported and explicitly satisfies the Kramers-Kronig relationship.

We plot in the inset in Fig. 1(a) the real part of the complex transverse dielectric susceptibility $\epsilon$ for both RADP:72 and RADP:35. The magnitude of $\epsilon^{\prime}$ along the $b$ axis scales roughly with $x$ in the paraelectric, with a gradual freezing over a similar temperature range. The main part of Fig. 1(a) presents the traditional signature of glassiness for RADP:72, a broad peak in $\epsilon^{\prime}(T)$ that narrows and moves to lower $T$ with decreasing $f$ [2]. However, there is far more information contained in these curves than the evolution of the peak in the spectrum. Taking cuts at constant $T$ and interrogating the system over many decades in $f$ [Figs. 1(b) and 1(c)] paints a more complete picture. The spectral response is enormously broad, with a decades wide rollover at higher $T$ assuming a strict logarithmic dependence on $f$ below $22 \mathrm{~K}$ for $x=0.72$ and $7 \mathrm{~K}$ for $x=$ 0.35 . A single Debye relaxation would produce a peak in $\epsilon^{\prime \prime}(f)$ with a full width at half maximum of 1.14 decades 


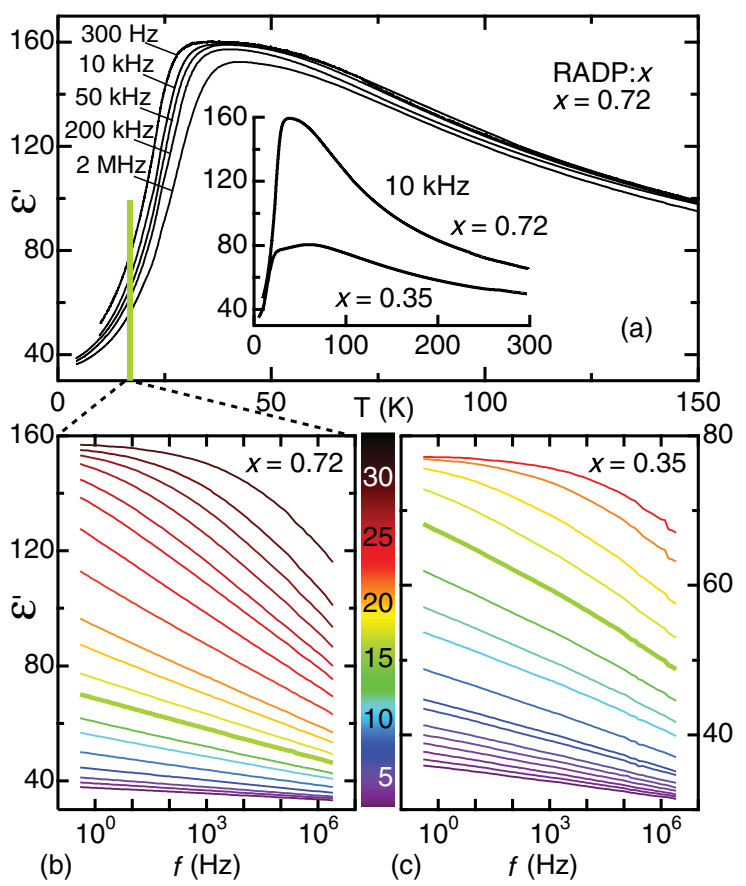

FIG. 1 (color). Dielectric susceptibilities of RADP as a function of $T, f$, and $x$. (a) Temperature dependence of $\epsilon^{\prime}$ for $a c$-cut RADP:72 at five different measurement frequencies. (Inset) The concentration dependence of the freezing at $f=10 \mathrm{kHz}$ for two RADP crystals with conjugate ratios of FE to AFE constituents. (b),(c) Slices at fixed temperature over seven decades in frequency reveals the spectral character of $\epsilon^{\prime}$ for RADP:72 and RADP:35, respectively. [ $T(\mathrm{~K})$ from top to bottom: (b) 29.9, 28.7, 27.6, 26.5, 25.4, 24.5, 23.4, 22.2 , 20.6, 19.4, 17.5, 15.7, 12.9, 10.6, $8.7,6.7,4.8,3.7,2.7$; (c) $23.2,20.9,18.9,17.2,15.4,12.8,11.4$, $10.3,8.5,7.0,6.5,5.5,4.9,4.2,3.7,3.2,2.7$.$] The logarithmic$ dependence at low $T$ is nearly identical for both crystals.

and a sharp rise in $\epsilon^{\prime}(f)$ to a frequency-independent value at low $f$. By contrast, the $\log f$ dependence represents fluctuations over a broad swath of times and corresponds to $1 / f$ noise in the polarization. Both the slope and the magnitude of the susceptibility become essentially independent of $x$ in this low $T$ regime, suggesting a local relaxation process.

A $\log f$ dependence picks out no characteristic frequency per se, but, as demonstrated in Fig. 2, the extrapolation of the data to higher frequencies defines a convergence point at $f_{0} \approx 10^{11} \mathrm{~Hz}$, independent of $T$. Although this is a large extrapolation, previous microwave studies of RADP [9] indicated that the logarithmic form extended from $f=10^{7}$ to $10^{10} \mathrm{~Hz}$, and previous infrared studies [10] bounded this frequency below $2 \times 10^{12} \mathrm{~Hz}$. Here again, we find the same $f_{0}$ for both RADP:35 and RADP:72. This can be seen most clearly (Fig. 2, inset) by the identical slopes for the two crystals in a plot of $\epsilon^{\prime}(f=1 \mathrm{~Hz})$ vs $\left|d \epsilon^{\prime} / d \log f\right|$.

In order to decipher the origin of $f_{0}$, we employed neutron Compton scattering (NCS) [3] to study local proton motion in the hydrogen-bond network. NCS experi-

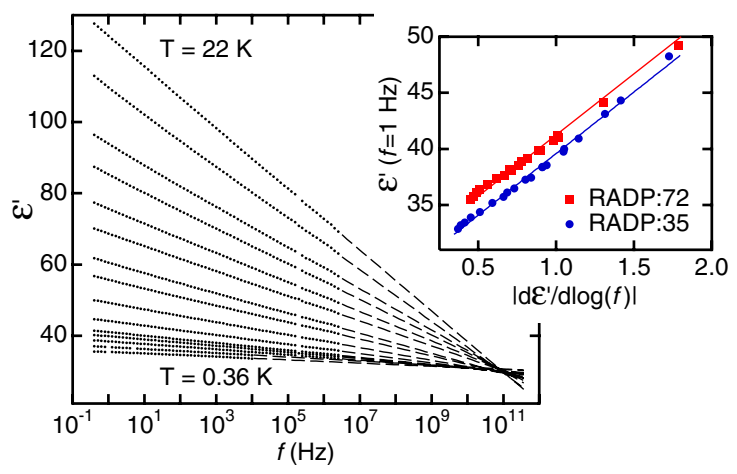

FIG. 2 (color online). Extrapolating the logarithmic dependence of $\epsilon^{\prime}$ for RADP:72 to higher $f$ (dashed line, $f>$ $2.5 \mathrm{MHz}$ ) defines a $T$-independent convergence point at $f_{0} \approx$ $10^{11} \mathrm{~Hz}$. (Inset) A plot of $\epsilon^{\prime}(f=1 \mathrm{~Hz}) \mathrm{vs}\left|d \epsilon^{\prime} / d \log f\right|$ indicates the same convergence point $f_{0}$ within error bars for both crystals $\left(9.6 \pm 4.0 \times 10^{10}\right.$ and $8.5 \pm 0.6 \times 10^{10} \mathrm{~Hz}$ for RADP:35 and RADP:72, respectively), indicating a local relaxation mechanism.

ments on RADP:72 and RADP:35 at $T=20 \mathrm{~K}$ were performed on Vesuvio at ISIS, the pulsed neutron source at the Rutherford Laboratory. For neutrons with kinetic energies of 5-100 eV, NCS occurs in a time scale of $10^{-15}-10^{-16} \mathrm{~s}$, permitting the capture of an instant image of the proton momentum distribution in the impulseapproximation limit. The inelastic scattering is entirely incoherent, and the dynamical structure factor $S_{M}(\vec{q}, \omega)$ relates the energy and momentum transfers to the momentum distribution of the protons $n(\vec{p})$ by $S_{M}(\vec{q}, \omega)=$ $\int n(\vec{p}) \delta\left(\hbar \omega-q^{2} / 2 M-\vec{p} \vec{q} / M\right) d \vec{p}$, where $M$ is the mass of the proton, $\vec{p}$ is the momentum of the proton, and $\vec{q}$ and $\hbar \omega$ are the momentum and energy transfers, respectively. For the measured $S_{M}(\vec{q}, \omega)$ within the $a b$ and $a c$ planes, a three-dimensional $n(\vec{p})$ was reconstructed. A cut of $n(\vec{p})$ along the bond axis is transformed to real space for construction of the proton potential [3]. For protons in the hydrogen bonds of RADP at $T=20 \mathrm{~K}$, the inversion revealed the double-well potential plotted in Fig. 3. The NCS technique is averaged, and, thus, the potential is necessarily symmetric.

By itself, the double-well structure is an indication that the proton is tunneling, because a proton localized at one position would reveal only a single well potential. The barrier width $d=0.33 \pm 0.02 \AA$ is very narrow and comparable with the $0.34 \AA$ found for the hydrogen bond in crystal KDP through neutron elastic diffraction [11]. The position of the ground-state level (Fig. 3) gives a barrier height $\Delta=58 \pm 10 \mathrm{meV}$. By comparison, the asymmetry of the potential was estimated to be $\sim 5 \mathrm{meV}$ [12]. Taken together, these parameters point to a large tunneling probability for a single proton. Within the WKB approximation, the quantum probability $w_{\mathrm{WKB}}=\exp \left(-2 d \sqrt{2 M_{p} \Delta} / \hbar\right)=$ 0.031, as compared to an Arrhenius thermal hopping 


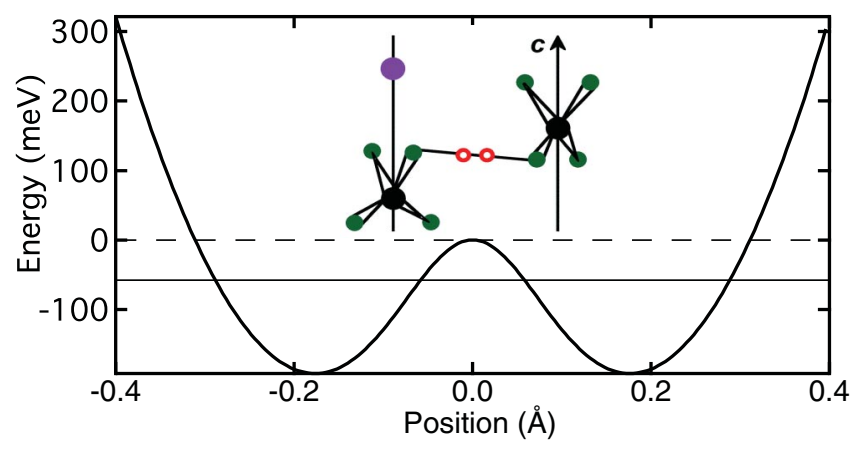

FIG. 3 (color). Local hydrogen-bond potential measured by neutron Compton scattering. The horizontal solid line marks the ground-state level relative to the top of the barrier (dashed line), calculated from the real-space double-well potential using an online software package [23]. (Illustration) Red rings represent the two possible positions for $\mathrm{H}$ on the hydrogen bond; green is $\mathrm{O}$; black is $\mathrm{P}$; purple is either $\mathrm{Rb}$ or the $\mathrm{NH}_{4}$ group (cf. Ref. [11]).

probability $\exp (-\Delta / k T)=3 \times 10^{-15}$ at $T=20 \mathrm{~K}$. The potential is not particularly sensitive to $x$, and it is even similar to that of KDP, supporting the notion that the local environment dominates the quantum relaxation at low $T$.

A single proton oscillating between two wells does not generate a polarization change and, hence, will not lead to dielectric relaxation. However, from Pauling's rule, protons interact with each other through the electrostatic force to maintain charge balance at each $\mathrm{PO}_{4}$ group. A simultaneous two-proton motion at each $\mathrm{PO}_{4}$ group generates a local electric dipole flip. There are two limiting cases: totally correlated tunneling of the protons with a mass twice that of a single proton and, at the other extreme, independent motion. The tunneling probability is then bounded by $w_{2 P}=\exp \left(-2 d \sqrt{2\left(2 M_{P}\right) \Delta} / \hbar\right)=7.2 \times 10^{-3}$ when correlated and $w_{2 P}=w_{\mathrm{WKB}}^{2}=9.3 \times 10^{-4}$ when uncorrelated. The single proton attempt frequency is calculated from the curvature about a minimum in the double well $f_{a}=\sqrt{M_{P}^{-1}\left|U^{\prime \prime}\left(x_{0}\right)\right|} / 2 \pi=7.2 \times 10^{13} \mathrm{~Hz}$, which is consistent with an optical phonon frequency [13] and supports the impulse approximation of NCS. Multiplying by the tunneling probabilities, we obtain a transition rate between $6.7 \times 10^{10}$ and $5.2 \times 10^{11} \mathrm{~Hz}$, which brackets our experimental result $f_{0} \approx 10^{11} \mathrm{~Hz}$.

This stochastic picture of collective proton motion near each $\mathrm{PO}_{4}$ group maps onto both the creation and the diffusive motion of defects, called Takagi configurations [14] (Fig. 4, inset), in the long-time limit with a dressed proton mass. Takagi configurations likely originate at local fluctuations in the FE-AFE competition [7], a type of frozen-in frustration with a constant number density below $22 \mathrm{~K}$ as demanded by the existence of the convergence point. Since RADP:72 and RADP:35 are nearly conjugate in concentration, the defect densities also should be similar. The fastest dielectric relaxation would be associated

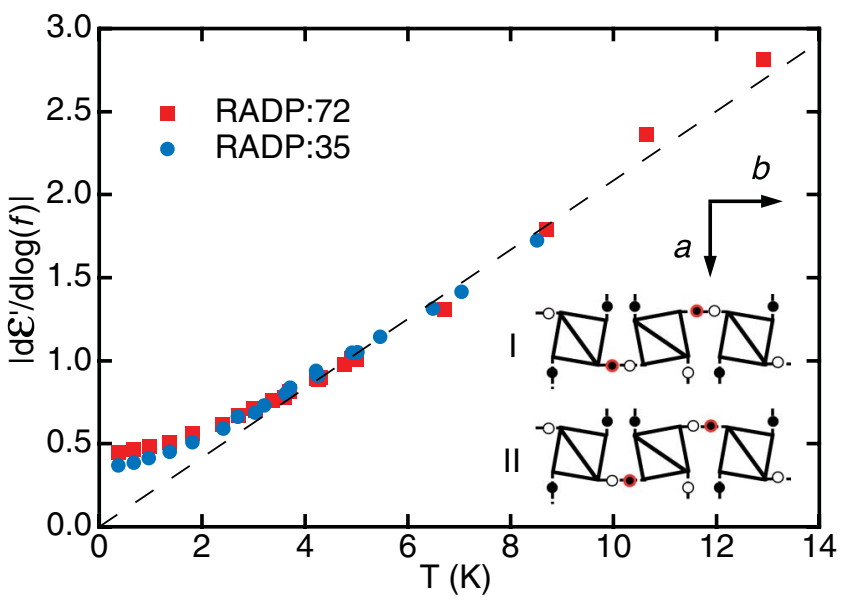

FIG. 4 (color). The logarithmic slope $\left|d \epsilon^{\prime} / d \log f\right|$ plotted as a function of $T$ indicates a classical to quantum crossover in the dielectric relaxation, similar for both crystals at $T=0$. (Inset) Takagi configuration, a type of defect where three protons (small solid circles) move close to $1 \mathrm{PO}_{4}$ group (e.g., top panel, left), instead of the two protons required for a Slater configuration (top panel, middle). The extra proton lets correlated protons (red pair) rearrange in the H-bond network. The two panels show the sequential movement of correlated protons projected onto the $a b$ plane. From a quantum-mechanical viewpoint, with a symmetric potential at least two protons must tunnel to create a stable new configuration.

with the local oscillation of a pair of correlated protons, while subsequently slower dielectric relaxation should be due to the diffusive propagation of Takagi configurations through the lattice structure. Macroscopic observables such as the dielectric susceptibility change when a Takagi configuration flips the local dipole moments associated with individual $\mathrm{PO}_{4}$ groups along its diffusive path, as illustrated in the inset in Fig. 4.

The low-frequency, long-time logarithmic relaxation of defects sequentially moving through pinning centers is reminiscent of glassy vortex relaxation in superconductors [15]. Pursuing this analogy leads to an electrical version of the magnetic Anderson-Kim model for thermally activated vortex relaxation [16]. For a polarization state $P$ in an electric field $E$, the total barrier a dipole moment $p$ faces is $U(P)=U_{0}+V_{0} E P / 2$, including a free energy barrier $U_{0}$ for the zero-field ground state and a self-energy term of the dielectric moment $p \approx P V_{0}$ in a volume $V_{0}$ containing roughly a segment of the Takagi configuration diffusion pathway. The dynamical equation for $P$ in the thermally activated regime,

$$
d P / d t \propto \exp (-U / k T)
$$

contains a potential linearly increasing with $P$, which gives a logarithmic dependence of $\epsilon^{\prime}$ on frequency [17]:

$$
\epsilon^{\prime}=\epsilon_{\mathrm{eff}}^{\prime}\left[1-\left(k_{B} T / U_{0}\right) \ln \left(f / f_{\text {eff }}\right)\right] \text {. }
$$


The effective attempt frequency $f_{\text {eff }}$ for the diffusion of Takagi configurations is the experimentally observed $f_{0}=$ $10^{11} \mathrm{~Hz}$. The measured $\epsilon^{\prime}\left(f_{\text {eff }}\right)$ includes contributions from both Takagi configuration diffusion and a background term from the electrons and heavy ions. The background ranges from 11 in the ordered AFE phase of RADP:90 [1] to 15 from infrared measurements of RADP:35 [18]. When the latter is subtracted, this gives a true $\epsilon_{\mathrm{eff}}^{\prime} \approx 15$.

We can use Eq. (2) to solve for key parameters that describe the free energy surface of the proton glass. We plot in Fig. $4\left|d \epsilon^{\prime} / d \log f\right|$ as a function of $T$ and, by taking the tangential slope (dashed line), find $U_{0} / k_{B}=$ $\epsilon_{\text {eff }}^{\prime} /\left[d\left(\left|d \epsilon^{\prime} / d \ln f\right|\right) / d T\right] \approx 165 \mathrm{~K}$. $U_{0}$ represents the average barrier height between two nearby polarization states on the free energy surface. For $T<3 \mathrm{~K}$, the data in Fig. 4 deviate from the classical, linear temperature dependence and approach a finite relaxation rate at $T=0$. This classical-quantum crossover-strikingly similar for the two RADP crystals - can be accommodated within the theory by recasting Eq. (1) with the Euclidean action $S$ [15]: $d P / d t \propto \exp (-S / \hbar)$. If the action also has a linear dependence on $P$, which should be true to first order because of the symmetry-breaking measurement field $E$, then the dielectric susceptibility retains its logarithmic dependence on $f$, with only $k_{B} T / U_{0}$ replaced by $\hbar / S$ :

$$
\boldsymbol{\epsilon}^{\prime}=\boldsymbol{\epsilon}_{\mathrm{eff}}^{\prime}\left[1-(\hbar / S) \ln \left(f / f_{\mathrm{eff}}\right)\right] \text {. }
$$

From the measured $T=0$ intercept of $\left|d \epsilon^{\prime} / d \log f\right|$ vs $T$, we can use Eq. (3) to evaluate the quantum action for a tunneling dipole moment, $S=\hbar \epsilon_{\text {eff }}^{\prime} /\left.\left(\left|d \epsilon^{\prime} / d \ln f\right|\right)\right|_{T=0} \approx$ $85 \hbar$. A first principles theory to test this experimental result for a fundamental quantum-mechanical parameter is difficult given the importance of dissipation for macroscopic systems [19], but we can bound the result from below via a dissipationless WKB approach. For a Takagi configuration with a two-proton mass $M_{T}$ to tunnel through an energy barrier $U_{0}$ of $170 \mathrm{~K}$, the WKB method estimates an action $S=2 d_{0} \sqrt{2 M_{T} U_{0}} \approx 54 \hbar$ over an instantaneous spatial distance $d_{0}=6.9 \AA$, which is the separation between next nearest neighbor $\mathrm{PO}_{4}$ groups (Fig. 4, inset). Both anisotropic diffusion of the Takagi configurations and dissipation would slow the relaxation and increase $S$, bringing the theoretical prediction closer to the experimental result.

The proton glass is cited as an experimental realization of the Ising model in a fixed transverse field [20]. We have described a quantitative picture of the dynamics from local proton motion in hydrogen bonds to the correlated movement of polarization states on the free energy surface. Given the piezoelectric nature of RADP, applying either a dc electric field or uniaxial stress can modify the free energy surface [21], effectively turning the transverse field into a continuous variable [22] and opening the possibility of new tests of quantum relaxation in correlated and glassy systems.

We thank G. Crabtree, Y. Galperin, R. Jaramillo, M.-L. Saboungi, G. Seidler, P. Simon, V. Vinokur, and especially S. Nagel for helpful discussions and T. Abdul-Redah, A. Bytchkov, J. Hu, J. Mayers, and D. Silevitch for technical assistance. Work at the University of Chicago was supported by the NSF MRSEC Grant No. DMR-0213745. G. R. acknowledges DOE No. 1-5-555229 for the NCS effort.

*Corresponding author.

Electronic address: tfr@uchicago.edu

[1] E. Courtens, Ferroelectrics 72, 229 (1987); U. T. Höchli, K. Knorr, and A. Loidl, Adv. Phys. 39, 405 (1990).

[2] K. Binder and A.P. Young, Rev. Mod. Phys. 58, 801 (1986).

[3] G. Reiter and R. Silver, Phys. Rev. Lett. 54, 1047 (1985); G. F. Reiter, J. Mayers, and P. Platzman, Phys. Rev. Lett. 89, 135505 (2002).

[4] G. A. Jeffrey, An Introduction to Hydrogen Bonding (Oxford University, New York, 1997).

[5] L. Pauling, The Nature of the Chemical Bond (Cornell University, Ithaca, 1960), 3rd ed.

[6] J. C. Slater, J. Chem. Phys. 9, 16 (1941).

[7] E. Courtens, T.F. Rosenbaum, S.E. Nagler, and P. M. Horn, Phys. Rev. B 29, 515 (1984).

[8] J.-F. Berret et al., Phys. Rev. Lett. 67, 93 (1991).

[9] H. J. Brückner, E. Courtens, and H.-G. Unruh, Z. Phys. B 73, 337 (1988).

[10] J. Petzelt et al., J. Phys. Condens. Matter 3, 2021 (1991); A. A. Volkov et al., Sov. Phys. JETP 74, 133 (1992).

[11] R. J. Nelmes, Z. Tun, and W. F. Kuhs, Ferroelectrics 71, 125 (1987).

[12] V.H. Schmidt, S. Waplak, S. Hutton, and P. Schnackenberg, Phys. Rev. B 30, 2795 (1984).

[13] E. Wiener, S. Levin, and I. Pelah, J. Chem. Phys. 52, 2881 (1970).

[14] Y. Takagi, J. Phys. Soc. Jpn. 3, 273 (1948); R. Kind et al., Phys. Rev. Lett. 88, 195501 (2002).

[15] G. Blatter, V.B. Geshkenbein, and V. M. Vinokur, Phys. Rev. Lett. 66, 3297 (1991); G. T. Seidler et al., Phys. Rev. Lett. 74, 1442 (1995); Y. Yeshurun, A. P. Malozemoff, and A. Shaulov, Rev. Mod. Phys. 68, 911 (1996).

[16] P. W. Anderson, Phys. Rev. Lett. 9, 309 (1962).

[17] A. P. Malozemoff, Physica (Amsterdam) 185C, 264 (1991).

[18] P. Simon, Ferroelectrics 135, 169 (1992).

[19] A. O. Caldeira and A. J. Leggett, Phys. Rev. Lett. 46, 211 (1981).

[20] R. Pirc, B. Tadic, and R. Blinc, Z. Phys. B 61, 69 (1985).

[21] G. A. Samara and H. Terauchi, Phys. Rev. Lett. 59, 347 (1987).

[22] J. Brooke, T.F. Rosenbaum, and G. Aeppli, Nature (London) 413, 610 (2001).

[23] http://fermi.la.asu.edu/Schroedinger/. 